\title{
Redes semánticas y mapas conceptuales: una experiencia en la formación de bibliotecarios
}

\author{
Miriam Vieira da Cunha \\ Edna Lúcia da Silva \\ Universidade Federal de Santa Catarina (Brasil)
}

\subsection{Resumen}

Reflexión sobre las experiencias de enseñanza con mapas conceptuales realizadas en el Curso de Biblioteconomía de la Universidade Federal de Santa Catarina (Florianópolis, Brasil). Los objetivos fueron trabajar la inclusión determinar la idea central del texto y los conceptos o términos que la representan estas ideas - ; la conexión - las relaciones posibles con conocimientos previos de los estudiantes -; la diferenciación - los significados que pueden ser atribuidos a los términos y conceptos en el contexto del texto-; la integración - relaciones entre los términos y conceptos principales y la red de relaciones establecidas por el autor con ellos en la construcción del texto-. El proceso de aprendizaje significa crear mecanismos para despertar en cada alumno sus potencialidades, sus intereses y su vocación. Compartir en clase las distintas "redes de significados" a través de los mapas conceptuales es una actividad que estimula el pensamiento reflexivo y la construcción del conocimiento. La educación en el siglo XXI debe ser un proceso para toda la vida. Deberá preocuparse de la formación del ciudadano, de la persona y no solo de su formación profesional. El uso de los mapas conceptuales es eficaz para despertar en los estudiantes la capacidad de situar la información en su contexto e integrarla en la realidad.

Palabras clave: Mapas conceptuales. Educación de Bibliotecarios. Brasil.

\subsection{Abstract}

Reflection on the educational experiences with conceptual maps in Librarianship Education carried at the Universidade Federal de Santa Catarina (Florianópolis, Brazil). The aim was using conceptual maps to improve certain cognitive abilities: inclusion - finding the central idea of a text-, connection -expressing relations with the previous knowledge of the students), differentiation - attributing meanings to terms and concepts in a due textual context - and integration - relations among the main terms and concepts - . The learning process requires the personal construction and appropiation of knowledge. The net

Scire. $10: 1$ (en.-jun. 2004) 159-168. 
of meanings established by the student while building conceptual maps allows him to construct his own personal knowledge step by step, establishing new relationships. Sharing in the classroom the different nets of meanings established by individual students through conceptual maps can stimulate the reflective thought and the construction of knowledge.

Keywords: Conceptual maps. Library Science education. Brazil.

\section{Introducción: una nueva realidad educacional}

La realidad que estamos viviendo, en este inicio de siglo, se caracteriza por transformaciones intensas y constantes. Este nuevo escenario demanda profesionales con capacidad para aprender continuamente. La actividad educacional, en este contexto, requiere nuevas posturas y nuevas formas de acción. Según Castaño (2001, p. 77) "se debe pensar en la formación de jóvenes con autonomía intelectual, con ardor por la búsqueda del conocimiento, con postura ética que los torne comprometidos con los destinos de la sociedad humana".

Ahora bien, la enseñanza nunca estuvo, como hoy, volcada hacia el desarrollo de competencias que posibiliten la autonomía de las personas, entendiendo por competencia, en el sentido de Le Boterf (apud Perrenoud, 2001), la facultad de movilizar un conjunto de recursos cognitivos (saber, capacidad, información, etc.) y afectivos para afrontar con pertinencia y eficacia una serie de situaciones. Como competencias fundamentales para la autonomía de las personas, Perrenoud (2000) identificó ocho grandes categorías: saber identificar, evaluar y valorar sus posibilidades, sus derechos, sus límites y sus necesidades; saber formar y conducir proyectos y desarrollar estrategias, individualmente o en grupo; saber analizar situaciones, relaciones y campos de fuerzas de manera sistémica; saber cooperar, actuar en sinergia, participar de una actividad colectiva y compartir liderazgo; saber construir y estimular organizaciones y sistemas de acción colectiva de tipo democrático; saber gestionar y superar conflictos; saber convivir con reglas, usarlas y elaborarlas; y saber construir normas negociadas de convivencia que se sobrepongan a las diferencias culturales. Para Perrenoud (1999), las competencias se expresan a través de acciones y no son, en sí mismas, conocimientos; sino que utilizan, integran, o movilizan tales conocimientos. Así, una competencia es más que la simple aplicación de conocimientos: Implica un análisis que permite evaluar la pertinencia de los conocimientos para una determinada situación y la movilización de éstos con discernimiento.

El centro del proceso productivo, en esta realidad, se disloca hacia el aprender a aprender o aprender a conocer. En este paradigma educacional el estudiante es el sujeto de su propio aprendizaje, en un proceso progresivamente más autónomo. Como afirman Ontoria Peña et al. (1996 p. 13) "pensar da autonomía, capa- 
cidad crítica y reflexiva, tolerancia y libertad". La persona que piensa de manera autónoma tiene capacidad de escoger y tomar decisiones por sí sola.

El espíritu humano, según Delacote (1996), trata los símbolos y las informaciones que recibe y crea operaciones para manipularlos y almacenarlos en la memoria. La estructura de almacenamiento de la información en la memoria es asociativa: las estructuras simbólicas representan agrupamientos de informaciones. Estos grupos se unen entre sí formando redes de informaciones asociadas. Las asociaciones se establecen cuando se aprende. Aprender es, por lo tanto, establecer comparaciones, hacer uniones. La capacidad de hacer asociaciones permite entender el sentido de las cosas, comprender el mundo, hacer previsiones e inferencias. Enseñar significa, desde este punto de vista, crear mecanismos para despertar en cada alumno sus potencialidades para aprender, sus intereses y su vocación. Significa recordar que cada ser humano posee vivencias, experiencias y conocimientos que deben servir de fundamento para nuevos aprendizajes. Dentro de esta visión y en un contexto de búsqueda de procesos pedagógicos innovadores, se realizó en la Universidad de Santa Catarina una experiencia de aplicación de mapas conceptuales como estrategia de aprendizaje.

\section{Los mapas conceptuales}

Los mapas conceptuales, según Novak (apud Ontoria Peña et al., 1996), son una proyección práctica de la teoría del aprendizaje significativo de David Ausubel. En la utilización de los mapas conceptuales como estrategia de enseñanza está subyacente un modelo de pedagogía no concentrada en el profesor, sino en el estudiante; que atiende al desarrollo de las competencias y no solo a la repetición de la información; y que pretende el desarrollo harmónico de todas las dimensiones del individuo y no solamente de las intelectuales. El aprendizaje significativo, según este autor (1963), es el mecanismo mental que permite adquirir y almacenar las ideas e informaciones representadas en cualquier campo del conocimiento. El aprendizaje significativo, en su concepción, tiene como características básicas la "no-arbitrariedad" y la sustantividad. "No arbitrariedad", para este autor, se refiere al hecho de que el material potencialmente significativo se relaciona de manera "no arbitraria" con los conocimientos ya existentes en la estructura cognitiva del aprendiz, llamados por Ausubel "subsumidores" (subsumers). Los "subsumidores" son, pues, conocimientos relevantes preexistentes en la estructura cognitiva. El conocimiento previo, sirve de matriz idealizadora y organizacional para la incorporación, comprensión y fijación de los nuevos conocimientos. Los "subsumidores" son el puntal en la estructura cognitiva para que ese proceso se realice. Por su parte, sustantividad significa que lo que es incorporado a la estructura cognitiva es la sustancia o contenido del nuevo conocimiento, de las nuevas ideas, y no las palabras precisas usadas para expresarlas. El mismo

Scire. $10: 1$ (en.-jun. 2004) 159-168. 
concepto o la misma proposición pueden ser expresados de diferentes maneras $\mathrm{y}$, debido a eso, el aprendizaje significativo no puede depender del uso exclusivo de determinados signos.

Moreira (1997) explica que, en esta concepción, la esencia del proceso del aprendizaje significativo ocurre en la relación "no-arbitraria" y sustantiva de ideas simbólicamente expresadas con algún aspecto relevante de la estructura de conocimiento del sujeto. Para el aprendiz, los significados de los contenidos potencialmente significativos emergen de esta interacción. Moreira (1997) resalta que es también en esta interacción cuando el conocimiento previo se modifica por la adquisición de nuevos significados. En la perspectiva ausubeliana, según este autor, el conocimiento previo es la variable más importante para el aprendizaje significativo. Según Ausubel (apud Souza, 2003, p.5) "el factor individual más importante que influye en el aprendizaje es lo que el estudiante ya sabe." Siendo así, "un aprendizaje significativo implica la asimilación de conceptos y proposiciones nuevas, mediante su inclusión en las estructuras cognitivas y referenciales simbólicas existentes". Este planteamiento teórico implica, en consecuencia, un cambio de postura del profesor desde una situación en la que el alumno era un "receptor" de la información en él "depositada" a una nueva en la que se asocia la reflexión al proceso de construcción del conocimiento tratando de dotar a los estudiantes con herramientas para la selección y estructuración de sus propios conceptos. El proceso de aprendizaje pasa por el conocimiento del conjunto de representaciones que cada uno posee sobre lo que va a aprender. Debe ser visto como una construcción y una reconstrucción del conocimiento por el estudiante, que atribuye significados y establece representaciones del conocimiento que recibe, desarrollando, de esta forma, su red de estructuras y de significados. Esta forma de aprendizaje debe llevar el estudiante a una constante reformulación de ideas, en un proceso que posibilite la creación de un ambiente de aprendizaje significativo, según entende Souza (2003).

El legado más importante de la obra de Ausubel, fue el de desplazar la mirada de los estudiosos hacia el alumno como sujeto de aprendizaje, y, en particular, hacia los conceptos preexistentes en el alumno como reguladores de su propio aprendizaje; una perspectiva que está en ruptura con las concepciones behavioristas hasta entonces predominantes. Además, los conceptos centrales de la teoría del aprendizaje significativo continuaron inspirando y alimentando, durante los años $80 \mathrm{y}$ 90, varios estudios, señaladamente en el ámbito de la enseñanza de las ciencias. (Cachapuz, 2000). Entre ellos destaca Novak (apud Moreira, 1997), que "adoptó" la teoría de Ausubel y, consecuentemente, el concepto de aprendizaje significativo, dándole nuevos significados y ampliando su ámbito de aplicación: En su teoría humanista de educación, la educación es un conjunto de experiencias cognitivas, afectivas y psicomotoras que, cuando se guían por la Teoría del Aprendizaje

Scire. $10: 1$ (en.-jun. 2004) 159-168. 
Significativo, conducen a la potenciación y engrandecimiento (empowerment) del educando, preparándolo para actuar en un mundo en constante cambio.

Para Novak (apud Moreira, 1997) cualquier evento educativo es una acción para cambiar significados (pensar) y sentimientos en el aprendiz y el profesor. Un evento educativo, según él, está siempre acompañado de una experiencia afectiva. La predisposición para aprender, presentada por Ausubel como una de las condiciones para el aprendizaje significativo, está, para Novak, íntimamente relacionada con la experiencia afectiva que el aprendiz tiene del proceso educativo. Su hipótesis es que, si la experiencia afectiva es positiva e intelectualmente constructiva, el aprendiz avanza en la comprensión; y, recíprocamente, si la sensación afectiva es negativa y genera sentimientos de inadecuación, el aprendiz no siente que está aprendiendo. Predisposición para aprender y aprendizaje significativo guardan entre sí una relación prácticamente circular: el aprendizaje significativo requiere predisposición para aprender y, a su vez, genera este tipo de experiencia afectiva.

Los mapas conceptuales, ideados por Novak, son representaciones gráficas que "tienen como objetivo representar relaciones significativas entre conceptos en la forma de proposiciones. Una proposición consiste en dos o más términos conceptuales unidos por palabras de manera que forman una unidad semántica" (Novak y Gowin, 1999, p. 31). Permiten llamar la atención sobre las ideas consideradas más importantes en un determinado contexto y establecer las relaciones entre ellas. En esencia, permiten establecer representaciones gráficas de los conceptos de un campo específico del conocimiento. La construcción de los mapas se produce de tal forma que las interacciones entre los conceptos se hacen evidentes. Cada concepto queda conectado a otros mediante proposiciones a través de frases simplificadas. Esta "red de significados" establecida por el estudiante le permite construir su conocimiento paso a paso, dinamizándolo, estableciendo nuevas relaciones y reflexionando sobre ellas en el momento en el que las construye. El acto de compartir en clase las distintas "redes de significados" presentadas en los mapas constituye una actividad de estímulo al pensamiento reflexivo y a la construcción del conocimiento. En la elaboración de los mapas conceptuales deben ser considerados los siguientes presupuestos (Novak y Gowin, 1999, p. 31, 33, 36, 40):

- "Los mapas conceptuales sirven para poner de manifiesto, tanto a los profesores como a los alumnos, el pequeño grupo de ideas claves en las que se deben concentrar para desarrollar una tarea de aprendizaje específica."

- Los mapas conceptuales pueden servir también como mapa visual de ruta, mostrando algunos de los trayectos que se pueden seguir para encadenar los significados de los términos en preposiciones." 
- En el proceso de elaboración de los mapas se pueden desarrollar nuevas relaciones conceptuales, especialmente las que hasta el momento no se habían considerado. Los alumnos y profesores descubren en la elaboración de mapas nuevas relaciones y, por tanto, nuevos significados (o por lo menos significados que ellos no poseían conscientemente antes de que elaborasen el mapa). "En este sentido, la elaboración de mapas de conceptos puede ser una actividad creativa y puede ayudar a fomentar la creatividad."

- Los mapas conceptuales son útiles como instrumento para negociar significados. "Aprender el significado de un determinado conocimiento implica dialogar, cambiar, compartir y, a veces, establecer compromisos." Ahora bien, conviene indicar que esto no se refiere a la acción de compartir el aprendizaje. El aprendizaje es una actividad que no puede ser compartida; pues es una cuestión individual. Los significados, por el contrario "pueden ser compartidos, discutidos, negociados y sujetos a consenso."

- La elaboración de mapas conceptuales fomenta el trabajo cooperativo entre estudiantes y el profesor. "Cuando los mapas son elaborados en grupos de dos o tres estudiantes, pueden desempeñar una función social útil y conducir a animadas discusiones en clase."

- "Los mapas ayudan al estudiante a hacer evidentes los conceptos claves o las proposiciones que debe aprender, sugiriendo además las relaciones entre el nuevo conocimiento y lo que él o ella ya saben. La mejor forma de facilitar el aprendizaje significativo de los estudiantes es ayudarlos explícitamente a que vean la naturaleza y el papel de los conceptos, así como las relaciones entre ellos, tal como existen en sus mentes y como existen 'fuera', en el mundo o en instrucciones escritas u orales. Esta es una idea simple pero profunda: Los estudiantes pueden tardar meses o años, en reconocer que lo que ellos oyen, ven, sienten o huelen depende en parte de los conceptos que ellos tienen en sus mentes. Este objetivo es fundamental en un programa destinado a ayudar a los estudiantes a que aprendan a aprender". En segundo lugar, los mapas son procedimientos que ayudarán a los estudiantes a extraer conceptos específicos del material estudiado."

\section{Una experiencia en el ámbito de la Biblioteconomía}

Durante los años 2002-2003 se realizó una experiencia de trabajo con mapas conceptuales desde la filosofía del aprendizaje significativo (Ausubel, 1968, Novak; Gowin, 1990) en el marco de la asignatura de Información Aplicada a la Biblioteconomía del primer semestre de la carrera de Biblioteconomía de la Universidad Federal de Santa Catarina (UFSC). Esta asignatura representa el primer contacto, la puerta de entrada del estudiante en el mundo de la

Scire. $10: 1$ (en.-jun. 2004) 159-168. 
Biblioteconomía y la Ciencia de la Información. Es la única asignatura del área impartida en el primer semestre. Pretende dar al estudiante una visión general e integradora de la carrera y de la profesión, contribuyendo, de esta manera, al desarrollo del pensamiento crítico e inquisitivo indispensable para la práctica profesional. Debería, por tanto, permitir al alumno entender la lógica de la carrera y de las diferentes asignaturas que la componen y, fundamentalmente, ayudarle a comprender el elemento principal con el que va a trabajar: la información. La asignatura tiene como objetivos específicos introducir al alumno en el universo de la Biblioteconomía y de la Ciencia de la Información, esto es, en el contexto de desarrollo y las teorías del área; discutir los conceptos básicos del área; y facilitarle la comprensión del papel del profesional bibliotecario en el contexto de la sociedad del conocimiento.

La aplicación de mapas conceptuales, en el contexto de esta asignatura, se utilizó en el análisis de textos y la representación de conceptos con el objetivo de trabajar cuatro procesos cognitivos básicos, a saber: a) la inclusión, o sea, determinar cuál es la idea central del texto y que conceptos o términos la representan; b) la conexión, o sea, establecer relaciones con las ideas previas de los estudiantes; c) la diferenciación, esto es, indicar los significados que pueden ser atribuidos a los términos y conceptos en el entendimiento de los estudiantes; y d) la integración, o sea, mostrar cómo están relacionados los términos y conceptos principales y cuál es la red de relaciones percibidas por los estudiantes entre los términos y conceptos usados por el autor en la construcción del texto.

Para realizar la experiencia se motivó a un promedio de cuarenta alumnos al final del semestre para que leyeran dos artículos seleccionados por el profesor. Dichos artículos fueron seleccionados por ser lo suficientemente generales para abarcar los principales conceptos del área y sintetizar, en la medida de lo posible, la materia estudiada durante el semestre. Se estimuló también a los estudiantes para que se organizaran en grupos de estudio para que experimentaran el efecto de la discusión y la negociación de los significados de los conceptos encontrados en los artículos. Para facilitar su trabajo y en general la operatividad del proceso, se les dieron consignas claras y se les solicitó que anotaran en tiras los principales términos o conceptos encontrados en el texto; identificaran los conceptos más generales, los intermedios y los específicos; construyeran el mapa intentando organizar los conceptos anotados en las tiras en una hoja; analizaran y discutieran las razones que motivaron la ubicación de los conceptos en el mapa; verificaran si los conceptos más generales podían ser identificados en la representación; representaran los conceptos intermediarios y específicos y trazaran líneas de unión; y etiquetaran las líneas de unión con palabras nexo para indicar como los conceptos se relacionan entre sí. 
En el proceso de elaboración de mapas de conceptos en grupos, siempre se produce mucha discusión y mucha negociación sobre los significados y la ubicación de los conceptos en el mapa. Los estudiantes solicitan, en esta etapa, que el profesor corrija el mapa conceptual elaborado por ellos. El profesor explica entonces que no existen mapas correctos o incorrectos, sino representaciones conceptuales significativas o no. Un mapa conceptual no es explicativo diferentemente de textos y otros materiales educativos. Ellos son proyectados para que sean explicados por quien los hace.

Una vez construidos los mapas conceptuales, los grupos fueron invitados a compartirlos con los demás. A raíz de ello, se pudo observar que, efectivamente, la construcción de cada mapa conceptual, aunque la fuente básica sea la misma, es única. Las relaciones lógicas establecidas son diferentes, pero válidas en la concepción de los grupos. Las concepciones son diferentes pues dependen de la estructura cognitiva de los estudiantes que formaban cada grupo. Ausubel (apud Farias, 1995, p, 47) "se refiere a la estructura cognitiva como el contenido total y organizado de ideas de un determinado individuo o, en el contexto del aprendizaje de ciertos temas, al contenido y a la organización de sus ideas en aquel área particular del conocimiento". En esta experiencia particular, el mapa conceptual de cada grupo, como actividad de aprendizaje significativo, representó, simbólicamente, las ideas relacionadas con los conocimientos relevantes previamente adquiridos y captados por los estudiantes durante el semestre lectivo.

La experiencia de uso de mapas conceptuales en el Curso de Biblioteconomía de la Universidad Federal de Santa Catarina puede considerarse positiva, pues los objetivos previstos fueron alcanzados. Los estudiantes demostraron que sabían seleccionar los conceptos principales de los textos y establecieron, de forma lógica, los encadenamientos entre los conceptos principales, intermedios y específicos en un lenguaje explícito y conciso. Los mapas se diferenciaban en detalles, pero todos contenían los conceptos claves de los textos. La experiencia demostró también que el $89 \%$ de los mapas eran concisos y mostraban las relaciones entre los conceptos principales de forma simple y visual.

La experiencia fue vivida de forma muy positiva por los estudiantes, según se puede evaluar a partir de sus propias declaraciones, algunas de las cuales se presentan a continuación:

Fue importante trabajar con mapas conceptuales, pues pude compartir mis experiencias de aprendizaje en el semestre. La identificación de los temas principales del texto y el establecimiento de relaciones permitió la fijación y la comprensión de los asuntos abordados.

La elaboración del mapa conceptual sirvió para aprender a organizar mejor los puntos clave de un asunto.

Scire. $10: 1$ (en.-jun. 2004) 159-168. 
Hacer el mapa conceptual fue una experiencia que permitió levantar las piezas fundamentales, que ayudaron a visualizar mejor los conceptos, las inclusiones y las posibilidades de análisis de un asunto.

La técnica del mapa conceptual permitió hacer el esquema de una determinada materia. Con este esquema resultó más simple su comprensión y, por haber sido elaborado mediante un razonamiento lógico y basarse en experiencias anteriores de aprendizaje, se facilitó el proceso de este nuevo aprendizaje.

El trabajo fue importante pues aprendí a organizar los temas tratados en los textos de forma lógica y a trabajar en grupo, discutiendo y analizando las relaciones entre los conceptos tratados en el texto y recordando los temas enseñados durante el semestre.

\section{Consideraciones finales}

Pensar y aprender son dos elementos de un mismo proceso y pueden realizarse de forma simultánea. En realidad, cuando pensamos, aprendemos; y cuando aprendemos, pensamos. Pensar significa analizar hechos, situaciones e ideas (pensamiento crítico) buscando soluciones alternativas y nuevas ideas (pensamiento creativo). Por su parte, para que se dé el aprendizaje significativo, según Ontoria Peña (1996), son necesarias tres condiciones: a) la información para el aprendizaje debe ser potencialmente significativa, o sea, debe permitir la construcción de significados; b) debe existir, en el estudiante, una disposición o motivación para aprender; y c) la disposición para aprender presupone una actitud activa.

La comprensión depende de la capacidad de establecer conexiones entre el conocimiento y las experiencias previos y nuevas ideas. En este sentido, la adquisición de nuevas informaciones supone su interacción con ideas preexistentes. Para Novak y Gowin (1999, p. 35) "los estudiantes necesitan practicar el pensamiento reflexivo del mismo modo que los equipos necesitan tiempo para practicar un deporte". El acto de hacer y de rehacer los mapas conceptuales y compartirlos con los demás puede ser considerado un "esfuerzo de grupo en el deporte de pensar".

En este tipo de aprendizaje, el profesor no es un trasmisor de conocimientos, sino un orientador; el instigador de un proceso que debe ser interactivo y permanente. A través del aprendizaje significativo el estudiante tiene un compromiso y una responsabilidad con el proceso de aprender. Esta forma de aprendizaje ocurre cuando el estudiante establece relaciones entre los nuevos conceptos o las nuevas informaciones y los conceptos y conocimientos que ya posee. Este aprendizaje se contrapone al "aprendizaje memorístico donde la información nueva no se asocia con los conceptos existentes en la estructura cognitiva, donde se produce una interacción mínima o nula entre la información recientemente adquirida y la información ya almacenada." (Ontoria Peña, 1996, p. 27). Los mapas conceptuales son instrumentos pedagógicos que auxilian en la formación de una "cabeza

Scire. $10: 1$ (en.-jun. 2004) 159-168. 
bien hecha”, porque permiten que los que los estudiantes, en vez de dedicarse a una acumulación pura y simple del saber, pasen a disponer de una "aptitud general para colocar y tratar problemas y principios organizadores que permiten unir los saberes y darles sentido" (Morin, 2000, p. 21). La educación, en el siglo XXI, debe ser un proceso para toda la vida. Debe preocuparse por la formación del ciudadano y de la persona y no solo de su formación profesional. Aprender a aprender es un jalón fundamental de ese camino.

\section{Referencias}

Ausubel, D. P. (1968). Educational psycology: a cognitive view. New York: Holt, Rinehart and Winston, 1968.

Cachapuz, A. F. (2000). A procura da excelência na aprendizagem. // Moreira, M. A.; Valadares, J. A.; Caballero, C.; Teodoro, V. D. (orgs.). Teoria da aprendizagem significativa. Peniche: III Encontro Internacional sobre Aprendizagem significativa, 2000. URL: <http://www.univ-ab.pt/cestudos/centros/cecme/ Peniche \%202000,\%20Teori a\%20da\%20Aprendizagem $\% 20$ Significativa, $\% 20$ Contributos $\% 20$ do\%20III\%20En contro\%20Internacional.pdf>. Consultado: 2003-08-25.

Delacote, G. (1996). Savoir apprendre: les nouvelles méthodes. Paris: Ed. Odile Jabob, 1996.

Faria, W. de. (1995). Mapas conceituais: aplicações ao ensino, currículo e avaliação. São Paulo: EPU, 1995.

Moreira, M. A. (1997). Aprendizagem significativa: um conceito subjacente. Burgos: Encontro Internacional sobre Aprendizagem Significativa, 1997. URL: <http:// www.if. ufrgs. br/ moreira/apsigsubport.pdf > . Consultado: 2003-08-25.

Morin, E. (2000). A cabeça bem-feita: repensar a reforma, reformar o pensamento. Rio de Janeiro: Bertrand Brasil, 2000.

Novak, J. D.; Gowin, D. B. (1999). Aprender a aprender. 2. ed. Lisboa: Plátano, 1999.

Ontoria Peña, A. et al. (1996). Los mapas conceptuales en el aula. Buenos Aires: Ed. Magisterio del Rio de La Plata, 1996.

Perrenoud, P. (2000). Construindo competências. // Nova Escola. São Paulo. 135 (set. 2000). URL: <http://novaescola.abril.com.br/>. Consultado: 2003-08-25.

Perrenoud, P. (1999). Construir as competências desde a escola. Porto Alegre: Artes Médicas Sul, 1999.

Perrenoud, P. (2001). Ensinar: agir na urgência, decidir na incerteza. 2. ed. Porto Alegre: Artmed, 2001.

Souza, R.R. (2000). Usando mapas conceituais na educação informatizada: rumo a um aprendizado significativo. 2000. URL: <http://www.edutec.net/Textos/Alia/MISC/ edrenato.htm>. Consultado: 2003-06-10. 